\title{
Polar Isodistance Curves on Parametric Surfaces
}

\author{
J. Puig-Pey, A. Gálvez, A. Iglesias* \\ Department of Applied Mathematics and Computational Sciences, University of \\ Cantabria, Avda. de los Castros, s/n, E-39005, Santander, Spain \\ \{puigpeyj, galveza, iglesias\}@unican.es
}

\begin{abstract}
In this paper, a new method for interrogation of parametric surfaces is introduced. The basic idea is to consider the distance measured on certain curves on a surface as an interrogation tool. To this aim, two different sets of characteristic curves are considered: the normal section curves and the geodesic curves. The differential equations of these sets of curves starting radially from a given point of the surface are stated. Then, they are solved numerically, introducing the arc-length on the surface as the integration variable. Associated with those curves we construct the polar isodistance curves which are obtained by joining the points at the same distance from a given point of the surface along the section or geodesic curves. Finally, some illustrative examples for NURBS surfaces, by far the most common surfaces in industry, are also described.
\end{abstract}

\section{Introduction}

An important and widely researched issue in CAGD (Computer-Aided Geometric Design) is that of surface interrogation. Roughly speaking, it consists of questioning geometrically understandable characteristics of already constructed surfaces. The surface interrogation techniques attempt to illuminate those characteristics that are not easily discernible by conventional rendering. Of course, many different methods can be applied to this purpose [1,2,4-6, 8, 19]. Among them, a very popular and interesting body of research is the so-called characteristic curves on a surface. These are curves reflecting either the visual or the geometric properties of the surface. For the visual properties we can use the reflection lines [7] and the isophotes [16], which help to evaluate the behavior and aesthetics of the surface under illumination models. On the other hand, the geometric properties can be accurately analyzed through the contour lines $[1$, 14, 17], the lines of curvature [1], geodesic paths [1, 12], asymptotic lines [19], etc.

A major advantage of the characteristic curves on a surface is that they do not depend on the surface parameterization. Unfortunately, for complicated surfaces (such as the NURBS surfaces) these curves can only be described as the solutions of ordinary differential equations (ODEs). Hence, working with these curves implies that those equations must be numerically solved.

* This research was supported by the CICYT of the Spanish Ministry of Education (projects TAP1998-0640 and DPI2001-1288) and the European Fund FEDER (Contract 1FD1997-0409).

P.M.A. Sloot et al. (Eds.): ICCS 2002, LNCS 2330, pp. 161-170, 2002.

(C) Springer-Verlag Berlin Heidelberg 2002 
On the other hand, the notion of distance on a surface has been applied to several machining problems. For example, it has been shown that there is no simple way to describe the guiding surface for the cutter of a five-axis machining tool [6]. In addition to some other standard techniques, curves of equal distance have been proposed for the tool paths. While avoiding the troublesome question of computing intersections [3,9] or offset curves [13], a procedure for easily calculating distances on a surface is absolutely necessary. Futhermore, the notion of distance plays a fundamental role in outstanding problems of $N C$ (numerical controlled) tool-path generation, namely the determination of the step-forward distance, the orientation of the tool or the interference checking between the tool and the surface.

In this paper, a combination of some characteristic curves and the distance on a surface is proposed as a new surface interrogation tool. Firstly, we introduce two new families of characteristic curves on a surface: the normal section curves and the geodesic curves. In our approach, both families of curves are assumed to start at a given point on the surface, which will usually be chosen by its relevance for interrogation. In both cases, the curves are obtained by solving systems of ordinary differential equations with their corresponding initial conditions and using parametric representations $(u(s), v(s))$, where $s$ is the arc-length parameter of the curve on the surface. Associated with the section and geodesic curves we define what we call polar isodistance curves which are obtained by joining the points at the same distance from a given point of the surface along the normal section or geodesic curves. These new curves will allow us to introduce the distance as a significant element for surface interrogation.

The structure of this paper is the following: in Section 2 some basic mathematical concepts to be used throughout the paper are introduced. Section 3 describes both the normal section curves and the geodesic curves. Section 4 discusses the numerical procedure to solve the systems of ODEs obtained in the previous section. Finally, Section 5 presents some examples of section and geodesic curves and their associated polar isodistance curves for the case of NURBS surfaces.

\section{Mathematical Preliminaries}

In this paper we restrict ourselves to the case of differentiable parametric surfaces. Therefore, they are described by a vector-valued function of two variables:

$$
\mathbf{x}(u, v)=(x(u, v), y(u, v), z(u, v)), \quad u, v \in \Omega \subset \mathbb{R}^{2}
$$

where $u$ and $v$ are the surface parameters. Expression (1) is called a parameterization of the surface $\mathbf{x}$. At regular points, the partial derivatives $\mathbf{x}_{u}(u, v)$ and $\mathbf{x}_{v}(u, v)$ do not vanish simultaneously. For $\left\{u=u_{0}, v=v_{0}\right\}, \mathbf{x}_{u}$ and $\mathbf{x}_{v}$ are vectors on the tangent plane to the surface at the point $\mathbf{x}\left(u_{0}, v_{0}\right)$, each being tangent to the parametric or coordinate curve $v=v_{0}$ and $u=u_{0}$, respectively. These vectors define the unit normal vector $\mathbf{N}$ to the surface at $\mathbf{x}\left(u_{0}, v_{0}\right)$ as: 


$$
\mathbf{N}=\frac{\mathbf{x}_{u} \times \mathbf{x}_{v}}{\left|\mathbf{x}_{u} \times \mathbf{x}_{v}\right|}
$$

where $\times$ denotes the cross product.

A curve in the domain $\Omega$ can be described by means of its parametric representation $\{u=u(t), v=v(t)\}$. This expression defines a three-dimensional curve on the surface $\mathbf{x}$ given by $\mathbf{x}(t)=\mathbf{x}(u(t), v(t))$. Applying the chain rule, the tangent vector of the curve $\mathbf{x}$ at a point $\mathbf{x}(t)$ becomes:

$$
\mathbf{x}^{\prime}(t)=\mathbf{x}_{u} u^{\prime}(t)+\mathbf{x}_{v} v^{\prime}(t)
$$

In this work the curve $\mathbf{x}$ will usually be parameterized by the arc-length $s$ on the surface. Its geometric interpretation is that a constant step $s$ traces a constant distance along an arc-length parameterized curve. Since some industrial operations require an uniform parameterization, this property has several practical applications. For example, in computer controlled milling operations, the curve path followed by the milling machine must be parameterized such that the cutter neither speeds up nor slows down along the path. Consequently, the optimal path is the one parameterized by the arc-length. In this case, the differential relation between $s, t, u$ and $v$ is given by the First Fundamental Form of the surface:

$$
s^{\prime}(t)^{2}=E u^{\prime}(t)^{2}+2 F u^{\prime}(t) v^{\prime}(t)+G v^{\prime}(t)^{2}
$$

where

$$
E=\mathbf{x}_{u} \cdot \mathbf{x}_{u} \quad, \quad F=\mathbf{x}_{u} \cdot \mathbf{x}_{v} \quad, \quad G=\mathbf{x}_{v} \cdot \mathbf{x}_{v}
$$

and "." indicates the dot product. For the sake of clarity, in this paper the parameter $s$ will be strictly used to refer to a curve parameterized by the arclength on the surface.

\section{New Characteristic Curves for Surface Interrogation}

\subsection{Section Curves}

Many authors have dealt with the problem of sectioning parametric surfaces $[3,6,9,10,14]$. This problem has several practical applications. For instance, the main goal of the contouring algorithms in NC tool-path generation is to generate ordered sequences of points on planar sections of the given surface, from which tool path approximations can be computed. In this section, a description of a planar section of a parametric surface in terms of ODEs is discussed. From here, the concept of polar isodistance section curve is introduced.

The differential equation of the section $\mathbf{x}(t)$ at a point $\mathbf{x}(u, v)$ of a parametric surface by a plane which is normal to a given vector $\mathbf{D}$ can be written as:

$$
\mathbf{x}^{\prime}(t) \cdot \mathbf{D}=0
$$

where, from (3) we obtain:

$$
\mathbf{x}_{u} \cdot \mathbf{D} u^{\prime}(t)+\mathbf{x}_{v} \cdot \mathbf{D} v^{\prime}(t)=0
$$


In this paper we do not consider singular cases; this implies that all the points on the section are regular points and that vector $\mathbf{D}$ is never normal to the surface along the section $\mathbf{x}(t)$.

Since a primary goal is to measure distances on curves on a surface, it is more interesting to consider the arc-length surface parameterization for Eq. (6). In this case, the corresponding differential equations for $\frac{d u}{d s}$ and $\frac{d v}{d s}$ are obtained from (4) and (6) as a system of first-order explicit ordinary differential equations:

$$
\begin{aligned}
& \frac{d u}{d s}= \pm \frac{\mathbf{x}_{v} \cdot \mathbf{D}}{\sqrt{E\left(\mathbf{x}_{v} \cdot \mathbf{D}\right)^{2}-2 F\left(\mathbf{x}_{u} \cdot \mathbf{D}\right)\left(\mathbf{x}_{v} \cdot \mathbf{D}\right)+G\left(\mathbf{x}_{u} \cdot \mathbf{D}\right)^{2}}} \\
& \frac{d v}{d s}=\mp \frac{\mathbf{x}_{u} \cdot \mathbf{D}}{\sqrt{E\left(\mathbf{x}_{v} \cdot \mathbf{D}\right)^{2}-2 F\left(\mathbf{x}_{u} \cdot \mathbf{D}\right)\left(\mathbf{x}_{v} \cdot \mathbf{D}\right)+G\left(\mathbf{x}_{u} \cdot \mathbf{D}\right)^{2}}}
\end{aligned}
$$

with the initial conditions:

$$
u(0)=u_{0} \quad, \quad v(0)=v_{0}
$$

associated with the point $\mathbf{P}=\mathbf{x}\left(u_{0}, v_{0}\right)$. Each solution of (7)-(8) defines a section curve $(u(s), v(s))$ in the parametric domain $(u, v)$ of the surface. The threedimensional section curve on the surface is given by $\mathbf{x}(u(s), v(s)$ ), which obviously passes through the point $\mathbf{P}$. In addition, it should be remarked that because $s$ is essentially a non-negative parameter, only two possible combinations for the signs in (7), namely $(+)$ with $(-)$ or alternatively $(-)$ with $(+)$, are allowed. Each of these feasible choices lead to a different solution for (7)-(8), associated with each of the pieces of the section curve starting from $\mathbf{P}$, joined with continuity at $\mathbf{P}$. Further, solving the system (7) for the same initial condition (that is, for the same point $\mathbf{P}$ ) and different directions on the tangent plane at $\mathbf{P}$ provides an interesting procedure for interrogation on the local behavior of the surface around this point. Such directions can be defined in terms of only one variable representing the angle between a partial derivative of the surface at $\mathbf{P}$, let us say $\mathbf{x}_{v}$, and the corresponding vector of direction. In the following, let $\mathbf{x}_{v}^{\perp}$ denote a vector of the tangent plane at a point $\mathbf{P}$ orthogonal to $\mathbf{x}_{v}$, which is given by:

$$
\mathbf{x}_{v}^{\perp}=-G \mathbf{x}_{u}+F \mathbf{x}_{v}
$$

These vectors $\mathbf{x}_{v}$ and $\mathbf{x}_{v}^{\perp}$ define an orthogonal reference system on the tangent plane at the point $\mathbf{P}$. Therefore, any unit vector $\mathbf{n}$ lying on such a tangent plane can be written as:

$$
\mathbf{n}=\cos (\alpha) \frac{\mathbf{x}_{v}}{\left|\mathbf{x}_{v}\right|}+\sin (\alpha) \frac{\mathbf{x}_{v}^{\perp}}{\left|\mathbf{x}_{v}^{\perp}\right|}
$$

where $\alpha$ represents the angle between $\mathbf{x}_{v}$ and $\mathbf{n}$. With this simple procedure, we can obtain the section of the surface by a plane containing the normal vector to the surface, $\mathbf{N}$ (see Eq. (2)), and whose intersection with the tangent plane at $\mathbf{P}$ is a line forming an angle $\alpha$ with the direction of $\mathbf{x}_{v}$ at $\mathbf{P}$. This section will be 
obtained by numerical integration of (7) and (8), where $\mathbf{n}$ is defined as in (10) and $\mathbf{D}$ is given by:

$$
\mathbf{D}=\mathbf{N} \times \mathbf{n}
$$

Varying this angle $\alpha$, a set of normal sections $\mathbf{x}(u(s), v(s))$ passing through $\mathbf{P}$ are calculated, all of them resulting from the rotation of the normal planes around the normal vector $\mathbf{N}$. Fixing now a value $s=s_{L}$ for the arc-length parameter $s$ and assuming that the trajectories have not reached the limits of the parametric domain of the surface, one might obtain the set of points at distance $s_{L}$ from $\mathbf{P}$ along all the section curves, defining a polar isodistance normal section curve associated with this value $s_{L}$. This curve can be understood as the polar representation of a pseudo circumference lying on the surface with center at $\mathbf{P}$ and radius $s_{L}$, which is measured from $\mathbf{P}$ to the points of the pseudo circumfererence along the normal section curves.

\subsection{Geodesic curves}

Given a curve $\mathbf{C}$ on a surface $\mathbf{x}$, its geodesic curvature at a point $\mathbf{P}$ is the curvature at this point of the projection curve of $\mathbf{C}$ on the tangent plane to $\mathbf{x}$ at $\mathbf{P}$. Geodesics are defined as the curves on a surface with zero geodesic curvature. It is well known that if a curve connects two points on a surface following a minimum length trajectory, then it must be a geodesic [18]. That makes the geodesics particularly attractive for surface interrogation, especially when distances measured along curves on the surface are considered. On the other hand, the geodesic curvature has been widely applied to surface interrogation [19]. Thus, the geodesics are an excellent tool to identify the boundaries between regions with positive and negative geodesic curvatures.

The geodesics of a parametric surface $\mathbf{x}(u, v)$ can be expressed through a system of two second-order ODEs [18] or alternatively as a system of four explicit first-order ODEs [1] given by:

$$
\left\{\begin{array}{l}
\frac{d u}{d s}=u^{\prime} \\
\frac{d v}{d s}=v^{\prime} \\
\frac{d u^{\prime}}{d s}=-\Gamma_{11}^{1} u^{\prime 2}-2 \Gamma_{12}^{1} u^{\prime} v^{\prime}-\Gamma_{22}^{1} v^{\prime 2} \\
\frac{d v^{\prime}}{d s}=-\Gamma_{11}^{2} u^{\prime 2}-2 \Gamma_{12}^{2} u^{\prime} v^{\prime}-\Gamma_{22}^{2} v^{\prime 2}
\end{array}\right.
$$

where $\Gamma_{i j}^{k}, i, j, k=1,2$ are the first kind Christoffel symbols which can be calculated from the $E, F, G$ coefficients of the First Fundamental Form of the surface (see Eq. (5)) and their first derivatives [18]. Once again, $s$ represents the arclength parameter on the surface such that the functions $u, v, u^{\prime}$ and $v^{\prime}$ depend solely on it. Observe again that the equations are formulated in the $u-v$ parameters and hence the integration of the system (11) will give a curve expressed as 
$(u(s), v(s))$ in the parametric domain $\Omega$ for $u, v$. Such a curve corresponds to a three-dimensional curve on the surface, given by $\mathbf{x}(u(s), v(s))$. The domain for the variable $s$ is an interval $[0, L], L$ being the length of the geodesic. The initial conditions for $u$ and $v$ can be easily established as:

$$
u(0)=u_{\text {in }} \quad, \quad v(0)=v_{\text {in }}
$$

where $\mathbf{x}\left(u_{i n}, v_{i n}\right)$ is the point $\mathbf{P}$ where the geodesic starts. On the contrary, the initial conditions for $u^{\prime}$ and $v^{\prime}$ require further discussion. Firstly, note that the relation $u^{\prime}(t)=c v^{\prime}(t)$ defines a direction on the tangent plane of the surface at a point $\mathbf{P}$. Indeed, inserting this relation in (3) we obtain

$$
\mathbf{x}^{\prime}(t)=c \mathbf{x}_{u} v^{\prime}(t)+\mathbf{x}_{v} v^{\prime}(t)=\left(c \mathbf{x}_{u}+\mathbf{x}_{v}\right) v^{\prime}(t)
$$

leading to the direction:

$$
\mathbf{d}=c \mathbf{x}_{u}+\mathbf{x}_{v}
$$

This direction $\mathbf{d}$ can also be characterized in terms of the arc-length by giving $u^{\prime}(s)$ and $v^{\prime}(s)$ in $\mathbf{P}$ for the curve $\mathbf{x}(s)=\mathbf{x}(u(s), v(s))$. Combining the previous relation $u^{\prime}(t)=c v^{\prime}(t)$ with (4) we obtain:

$$
u^{\prime}(s)=\frac{ \pm c}{\sqrt{E c^{2}+2 F c+G}} \quad, \quad v^{\prime}(s)=\frac{ \pm 1}{\sqrt{E c^{2}+2 F c+G}}
$$

Note also that, in order for the relation $u^{\prime}(t)=c v^{\prime}(t)$ to hold, the same sign must be taken in (14) for both $u^{\prime}(s)$ and $v^{\prime}(s)$. On the other hand, different values of the parameter $c$ are associated through (13) with different tangent directions $\mathbf{d}$ at $\mathbf{P}$. It should be remarked, however, that there is not a direct geometric interpretation of the values of this parameter $c$. Fortunately, a description of $c$ in terms of the angle $\alpha$ can be easily derived. From (9) and (10), the vector $\mathbf{n}$ can also be written as:

$$
\mathbf{n}=-\sin (\alpha) \frac{G}{\left|\mathbf{x}_{v}^{\perp}\right|} \mathbf{x}_{u}+\left(\frac{\cos (\alpha)}{\left|\mathbf{x}_{v}\right|}+\frac{F \sin (\alpha)}{\left|\mathbf{x}_{v}^{\perp}\right|}\right) \mathbf{x}_{v}
$$

For the vectors $\mathbf{d}$ and $\mathbf{n}$ to be parallel, it is enough to establish the proportionality of the coefficients of $\mathbf{x}_{u} \mathbf{y} \mathbf{x}_{v}$ in (13) and (15). From the corresponding equation, we obtain

$$
c=\frac{-\sin (\alpha) G\left|\mathbf{x}_{v}\right|}{\cos (\alpha)\left|\mathbf{x}_{v}^{\perp}\right|+\sin (\alpha) F\left|\mathbf{x}_{v}\right|}
$$

This equation allows us to rewrite a direction $\mathbf{d}$ on the tangent plane in terms of the angle $\alpha$, thus gaining a geometrical insight. Now, the following initial conditions for $u^{\prime}$ and $v^{\prime}$ are considered:

$$
u^{\prime}(0)=\left(\frac{d u}{d s}\right)_{s=0}=u_{i n}^{\prime} \quad, \quad v^{\prime}(0)=\left(\frac{d v}{d s}\right)_{s=0}=v_{i n}^{\prime}
$$


where $u_{i n}^{\prime}, v_{i n}^{\prime}$ are obtained from (14) and (16) by giving the starting angle $\alpha$ between the geodesic trajectory to be calculated and vector $\mathbf{x}_{v}$ at the initial point $\mathbf{P}=\mathbf{x}\left(u_{i n}, v_{i n}\right)$.

Analogously to the comments given for the normal sections, one can choose a set of initial angles $\alpha$ from the initial point $\mathbf{P}$. The integration of the system (11)-(12)-(17) allows us to construct the corresponding set of geodesics starting from $\mathbf{P}$ and forming such initial angles with vector $\mathbf{x}_{v}\left(u_{i n}, v_{i n}\right)$ at $\mathbf{P}$. The concept of polar isodistance curve also extends to geodesics: a fixed value $s=s_{L}$ for the arc-length parameter $s$ yields a set of points at distance $s_{L}$ from $\mathbf{P}$ along all the geodesic curves, defining a polar isodistance geodesic curve associated with this value $s_{L}$. The meaning of this curve is very similar to that of the section curve: due to the minimal distance property of geodesics, a polar isodistance geodesic curve for a given value $s=s_{L}$ is the geometric place of points on the surface which are at a minimum distance $s_{L}$ from $\mathbf{P}$, measured on the surface.

\section{Numerical Process}

Although (7)-(8) and (11)-(12)-(17) are both systems of first-order ODEs, the calculation of the analytical expression for $u$ and $v$ (and subsequently for $\mathbf{x}(u, v)$ ) as functions of the parameter $s$ can be, in general, a very hard task, if not impossible, which is beyond the scope of this work. Hence, to deal with the most typical surfaces in design, such as the NURBS surfaces (see [15] for a description), we are forced to use numerical techniques.

Fortunately, both systems of equations can be numerically integrated by applying some standard techniques. In particular, all the numerical work has been performed by using Runge-Kutta methods with the ODE solvers of Matlab [11]. It should be noticed, however, that when the surface consists of several patches (for example, the piecewise bicubic splines or the NURBS surfaces) some kind of continuity conditions must be imposed to assure that the differential model is still valid in the neighbourhood of the patch boundaries. On the contrary, the model must be strictly applied inside each patch treating it separately and reconstructing the initial conditions when the considered trajectory crosses from one patch to the next.

On the other hand, the domain $\Omega$ of the parametric surface should be taken into account during the integration process. The parametric domain of the most typical surfaces in design exhibits a rectangular structure $[2,6]$, whose boundaries impose additional restrictions to the integration method. In particular, we must check whether the trajectories reach the limits of the parametric domain. For example, in the case of nonperiodic NURBS surfaces [15], these intervals are limited by the first and last values of the corresponding knot vectors for $u$ and $v$. On the contrary, in the case of working with surfaces which are partially or completely closed, additional tests for self-intersection must be performed.

The procedure for building a polar isodistance normal section or geodesic curve associated with a given distance $s_{L}$ with respect to a reference point $\mathbf{P}$ on a surface consists of making a loop giving regularly-spaced $\alpha$ values for the initial 
directions from $\mathbf{P}$. Then, a step-by-step integration is performed for each normal section or geodesic trajectory, until the value $s=s_{L}$ is attained. The transversal isodistance curve is obtained by joining the end points, one for each normal section or geodesic, in the $(u, v)$ domain and obtaining its three-dimensional image on the surface.

\section{Some Illustrative Results}

In this section the performance of the proposed method is discussed by means of some illustrative examples from the NURBS surface family [15]. In general, the method deals with any parametric surface. However, because of their advantages in industrial environments, their flexibility and the fact that they can represent well a wide variety of shapes (such as conics and quadrics), we will focus here on NURBS surfaces.

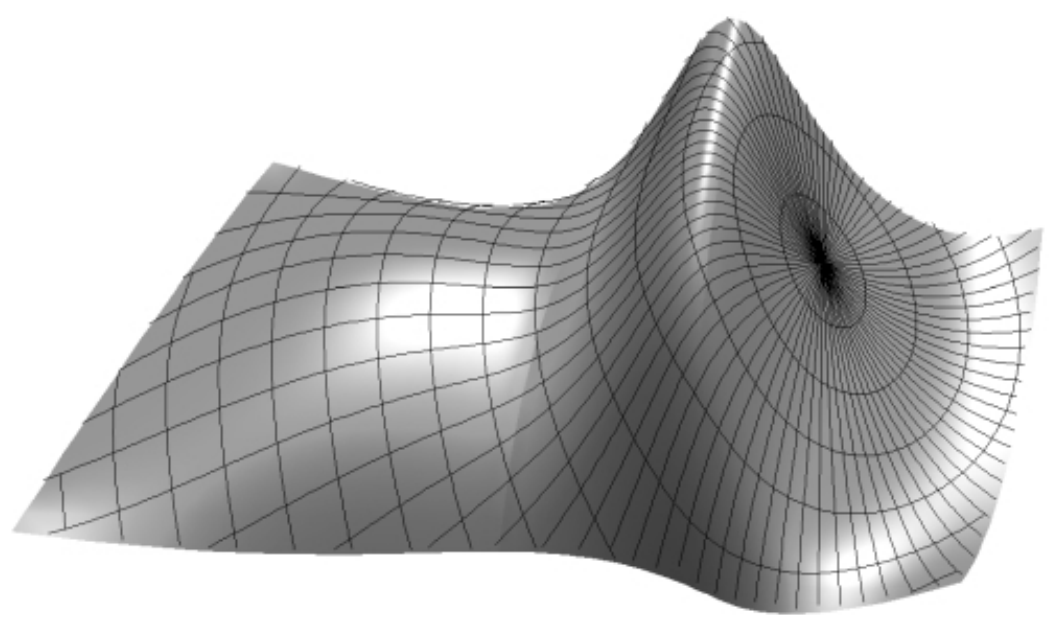

Fig. 1. Section curves and associated polar isodistance curves for a NURBS surface

As a first illustration, Fig. 1 shows a set of 80 normal section curves (obtained following Sec. 3.1) starting from a given point on a $(4,4)$-order NURBS surface defined by a grid of $4 \times 6$ control points and nonperiodic knot vectors for both $u$ and $v$. The surface has a single patch in the direction $u$ and three patches in the direction $v$. The figure also shows the polar isodistance normal section curves associated with different values for $s_{L}$ from 0.3 to 4.5 with step 0.3 . Of course, if the chosen distance $s_{L}$ always leads to points inside the domain of the surface, the polar isodistance curve will be closed. In this case, we can generate a continuous representation by simply interpolating the calculated points with a closed cyclic cubic spline, thus obtaining its image on the surface $\mathbf{x}(u, v)$. In 


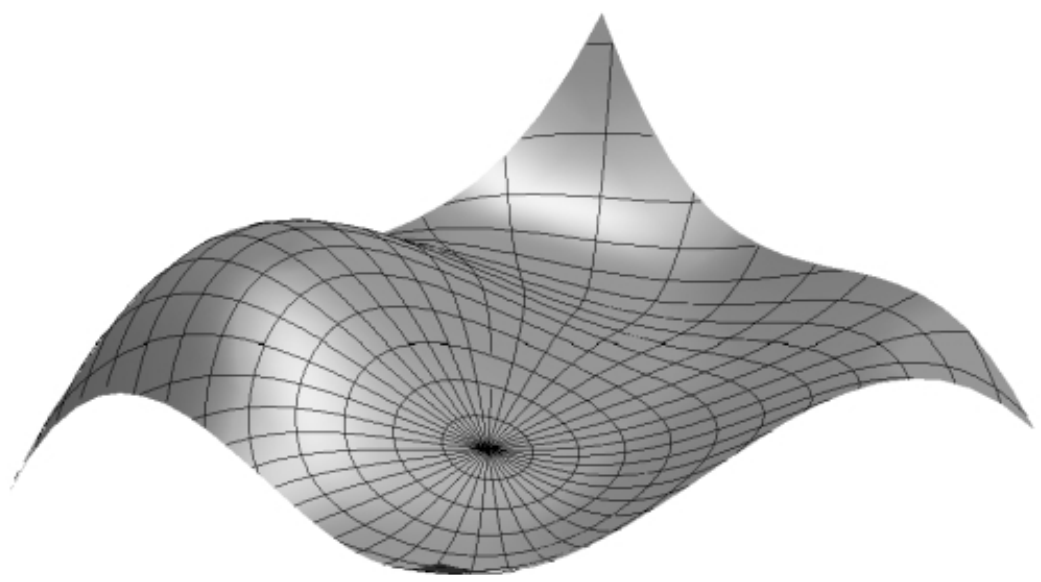

Fig. 2. Geodesic curves and associated polar isodistance curves for a NURBS surface

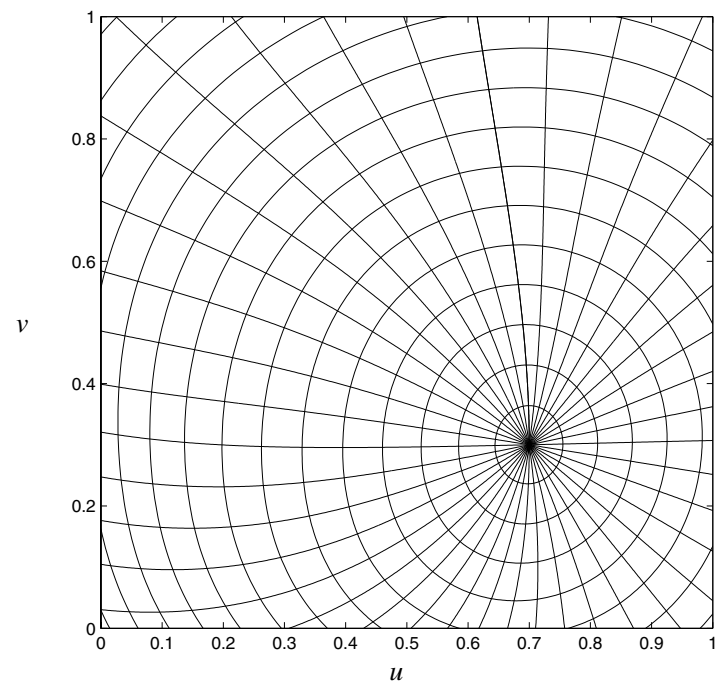

Fig. 3. Geodesic curves and associated polar isodistance curves in the $u-v$ parametric domain for the NURBS surface in Fig. 2

the present example, this only happens for $s_{L}=0.3$ and $s_{L}=0.6$. On the contrary, the curves will be made of unconnected pieces and only a continuous approximation of each piece can be obtained (for example, by using open cubic splines with the points calculated in the integration process).

As the second example, Fig. 2 shows a set of 40 geodesic curves (obtained following Sec. 3.2) referring to the point given by $\mathbf{x}(u=0.7, v=0.3)$ of a single- 
patch $(4,4)$-order NURBS surface defined by a grid of $4 \times 4$ control points as well as the polar isodistance geodesic curves associated with the values for $s_{L}$ from 1.25 to 20 with step 1.25. These geodesic curves and their corresponding polar isodistance curves are displayed in the surface parametric domain $\Omega$ in Fig. 3 .

\section{References}

1. Beck, J.M., Farouki, R.T., Hinds, J.K.: Surface analysis methods. IEEE Computer Graphics and Applications, Dec. (1986) 18-36

2. Farin, G.E.: Curves and Surfaces for Computer-Aided Geometric Design, 5th ed. Morgan Kaufmann, San Francisco (2001)

3. Grandine, T.A., Klein, F.W.: A new approach to the surface intersection problem. CAGD 14 (1997) 111-134

4. Hagen, H., Schreiber, T., Gschwind, E.: Methods for surface interrogation. Proc. Visualization'90, IEEE Computer Society Press, Los Alamitos, Calif. (1990) 187193

5. Hagen, H., Hahman, S., Schreiber, T., Nakajima, Y., Wördenweber, B., HollemannGrundstedt, P.: Surface interrogation algorithms. IEEE Computer Graphics and Applications, Sept. (1992) 53-60

6. Hoschek, J., Lasser, D.: Computer-Aided Geometric Design, A.K. Peters, Wellesley, MA (1993)

7. Klass, R.: Correction of local surface irregularities using reflection lines. CAD, 12(2) (1980) 73-77

8. Koenderink, J.J., van Doorn, A.J.: Surface shapes and curvature scales. Image and Vision Computing, 8(2) (1992) 557-565

9. Kriezis, G.A., Patrikalakis, N.M., Wolter, F.E.: Topological and differential equation methods for surface intersections. CAD, 24(1) (1992) 41-55

10. Lee, R.B., Fredricks, D.A.: Intersection of parametric surfaces and a plane. IEEE Computer Graphics and Applications, Aug. (1984) 48-51

11. The Mathworks Inc: Using Matlab. Natick, MA (1999)

12. Munchmeyer, F.C., Haw, R.: Applications of differental geometry to ship design. In: Computer Applications in the Automation of Shipyard Operation and Ship Design IV, Rogers, D.F., et al. (eds.) North Holland, Amsterdam (1982) 183-188

13. Patrikalakis, N.M., Bardis, L.: Offsets of curves on rational B-spline surfaces. Engineering with Computers, 5 (1989) 39-46

14. Petersen, C.S.: Adaptive contouring of three-dimensional surfaces. CAGD 1(1) (1984) 61-74

15. Piegl, L., Tiller, W.: The NURBS Book, Springer Verlag, Berlin Heidelberg (1997)

16. Poeschl, T.: Detecting surface irregularities using isophotes. CAGD 1(2) (1984) 163-168

17. Satterfield, S.G., Rogers, D.F.: A procedure for generating contour lines from a B-spline surface. IEEE Computer Graphics and Applications, Apr. (1985) 71-75

18. Struik, D.J.: Lectures on Classical Differential Geometry, 2nd ed., Dover Publications, New York (1988)

19. Theisel, H., Farin, G.E.: The curvature of characteristic curves on surfaces. IEEE Computer Graphics and Applications, Nov./Dec. (1997) 88-96 